\title{
Determining the Optimal Sleep Pattern to Promote Glymphatic Clearance of Amyloid-Beta in Individuals at Risk for Alzheimer's Disease: A Research Protocol
}

\author{
Muskan Bansal, BSc Student [1]*, Shahad S. Musa, BSc Student [1,2], Manitha Mulpuru, BSc \\ Student [2]
}

[1] Center for the Study of Biological Complexity, Virginia Commonwealth University, Richmond, Virginia, 23220

[2] Department of Biology, Virginia Commonwealth University, Richmond, Virginia, 23220

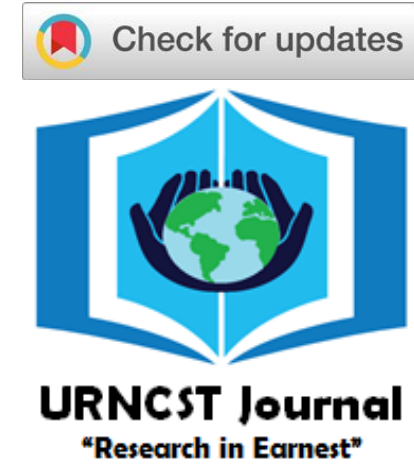

*Corresponding Author: bansalm@vcu.edu

\begin{abstract}
Introduction: Neurodegenerative disorders such as Alzheimer's disease (AD) are increasingly prevalent amongst older populations. Sleep irregularities are one of the chief complaints in people diagnosed with sleep disorders. Sleep has been hypothesized to ensure metabolic homeostasis and remove neurotoxic waste. Inadequate sleep leads to an accumulation of Amyloid-Beta $(\mathrm{A} \beta)$ levels which is associated with neurodegenerative diseases including AD and other dementias. Sleep is the driver of the glymphatic system, located in the perivascular space. The glymphatic system eliminates soluble proteins, including $A \beta$, from the central nervous system; transporting cerebrospinal fluid through the brain; and distributing macromolecules across the brain. This research proposal aims to identify optimal sleep patterns that decrease neurodegeneration by increasing glymphatic system functioning.

Methods: This study presents an ad-hoc study conducted over a 6-month period utilizing participants who have reported a family history of AD. Aß accumulation and cortisol levels will be measured to identify signs of neurodegeneration using brain scans, such as Positron Emission Tomography, and blood tests. Other parameters such as memory, energy rating, and sleep latency will be measured.

Results: Reduced slow-wave sleep may lead to a disruption in the glymphatic system. Monophasic sleep is said to have the most slow-wave sleep, and least rapid eye movement sleep which is most similar to wakefulness. Therefore, we hypothesize that monophasic sleep will slow neurodegeneration compared to biphasic and polyphasic sleep, contrary to popular belief that biphasic sleep is more beneficial than monophasic sleep.

Discussion: In this experiment, we expect to see increased glymphatic clearance after monophasic sleep. There are many factors that can impact sleep patterns and glymphatic clearance including work style, environment, culture, race, sex, and genetic markers for AD.

Conclusion: Further research that applies this suggested methodology should account for these variabilities when making conclusions on the optimal sleep pattern for plaque clearance in the brain. This proposal may improve research on AD by identifying the effects different sleep patterns have on the brain and neurodegeneration. Future research may study changes in sleep habits as a preventative measure for individuals who are at risk for or have been diagnosed with AD.
\end{abstract}

Keywords: Alzheimer's disease; sleep; neurodegeneration; glymphatic system; amyloid-beta

\section{Introduction}

Alzheimer's disease (AD) currently affects over 40 million people across the world, and with limited treatment options, its prevalence continues to increase. Although current treatment options serve to benefit the quality of life of those with the disease, they do not decrease the rate of decline. This neurodegenerative disease leads to memory loss, eventually affecting a person's ability to complete basic daily tasks. This disease can also lower the quality of life and increase financial burden on families. AD is ranked as the sixth leading cause of death in the United States and is the most common cause of dementia. Investigating daily life processes that can potentially impact the development of $\mathrm{AD}$ can lead to providing better prognosis tools for those who are at risk for AD.

A daily life process that has associations with $\mathrm{AD}$ is sleep. Sleep plays a critical role in the development and progression of neurological disorders including AD. Lack of sleep is considered an early marker for neurodegeneration. Neurodegeneration further amplifies fragmentation of the sleep-wake pattern by increasing sleep in the daytime and the frequency in nocturnal awakenings, worsening the disease state [1]. Disturbances in sleep, such as insomnia or hypersomnia, are associated with behavioral 
UNDERGRADUATE RESEARCH IN NATURAL AND CLINICAL SCIENCE AND TECHNOLOGY (URNCST) JOURNAL Read more URNCST Journal articles and submit your own today at: https://www.urncst.com

issues in $25-40 \%$ of individuals with mild to moderate dementia due to $\mathrm{AD}[2,3]$. While the disease state worsens, characterized by increased physical dependence on others and rapid cognitive decline, neurodegeneration and sleep decline also worsen [2]. Therefore, this feedforward loop exacerbates the neurodegeneration of the individuals. Understanding sleep as a daily process is pertinent to identifying ways to hinder the rapid development of the disease.

Recent research has proposed that a fundamental function of sleep is to establish a state for the glymphatic system to efficiently clear the central nervous system (CNS) of metabolic waste. It has been suggested that the glymphatic system is $90 \%$ more active during both natural and anesthetized sleep [4]. The brain must divide its resources between processes during the awake state, such as processing sensory information, and glymphatic clearance; therefore, there is increased cerebrospinal fluid (CSF) flow into the brain during sleep rather than during the awake state [5]. The glymphatic system functions during the sleep cycle and becomes largely disengaged during the wake cycle. Research also indicates that there is less arousal of the thalamus and the cerebral cortex during sleep, and therefore, less activity in the brain may also contribute to lower levels of metabolic waste [6]. Inadequate sleep thus leads to a decrease in the glymphatic system, waste removal, and transport of key compounds. The glymphatic system has been cited to play a role in reducing neurodegeneration, traumatic brain injury, and the onset of diseases.

The glymphatic system serves to clear cellular waste from the brain connecting both the perivascular network for CSF transport and the lymphatic network associated with the meninges, cranial nerves, and large vessels exiting the brain $[7,8]$. The driving force of the movement of the CSF pulls the interstitial fluid (ISF) into the perivenous space enabling the interchange between the CSF and ISF - the main component of the glymphatic system (Figure 1). Natural sleep or anesthesia have been associated with approximately a $60 \%$ increase in interstitial space and therefore, increased interchange between CSF and ISF leading to increased functioning of the glymphatic system. In a healthy brain, CSF flow is aided by highly polarized aquaporin-4 (AQP4) water channels on the astrocytic endfeet [9]. AQP4 facilitates the transport of CSF to the parenchyma, the functional portion of the brain. Dysfunction of AQP4 channels leads to a disruption of CSF flow, breakdown of the blood-brain barrier, brain inflammation, and the accumulation of metabolic protein waste in the glymphatic network. One common pathology of $\mathrm{AD}$ is the accumulation of a metabolic protein, AmyloidBeta $(A B)$, in the brain. $A B$ is formed from the proteolysis of a single-pass transmembrane protein known as amyloidbeta precursor protein (APP). This protein is metabolized rapidly and plays an important role in neuronal growth and repair. Accumulation of $A B$ is likely a result of decreased
APP metabolism or Aß clearance [10]. Mice model studies have shown that a lack of sleep in mice increase $A \beta$ accumulation [11,12]. This suggests a feedforward loop where the increase of $A B$ is associated with a lack of sleep, both of which may worsen AD symptoms.

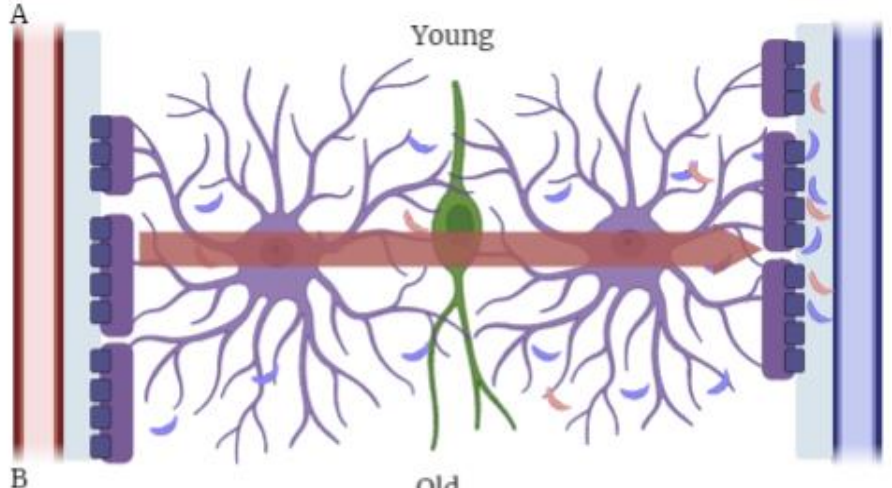

B

old

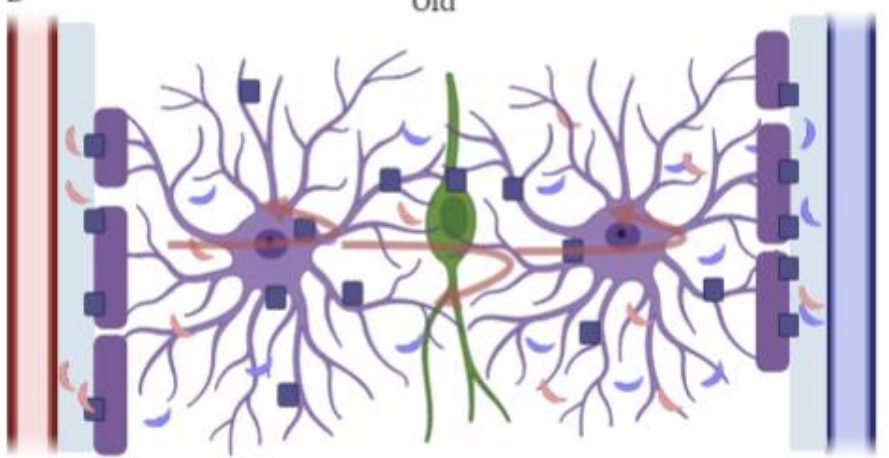

C Alzheimer's Disease

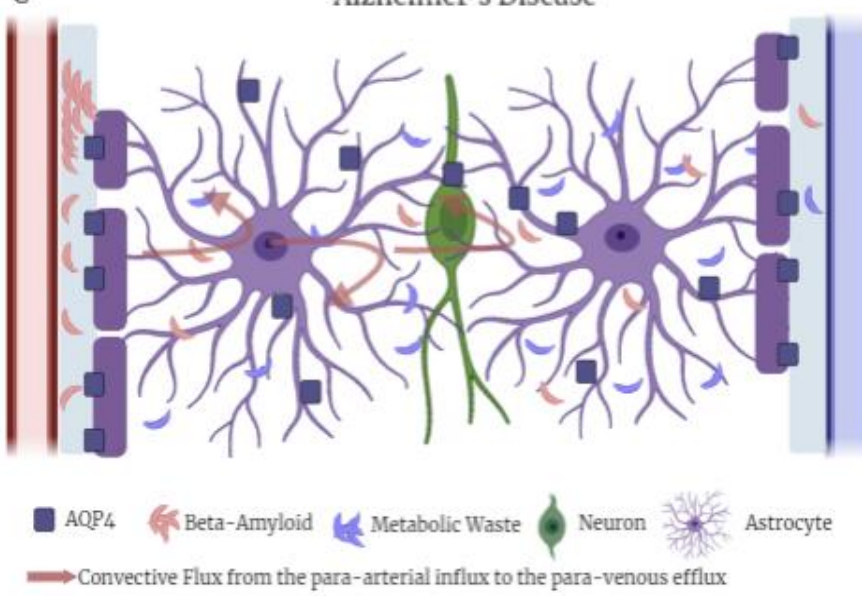

Figure 1. Amyloid-beta clearance via the glymphatic system in young adults, older adults, or those with AD. In younger individuals, AQP4 remains highly polarized on the vascular end-feet. As individuals age, AQP4 begins losing its polarity and $A ß$ accumulates in the perivascular space. This worsens in individuals with $\mathrm{AD}$, and $\mathrm{A} B$ plaque builds up blocking para-arterial influx. This allows more $A B$ and metabolic waste to enter the perivascular space, ultimately decreasing glymphatic clearance. This figure was created using Biorender. 
Identifying sleep patterns that may reduce $A ß$ accumulation could slow down cognitive decline and disease progression in individuals with AD.

It is well known that humans have many different sleep patterns, which are thought to arise from variability in environmental conditions such as warmer climates and industrialization [13]. Research has demonstrated three different types of sleep patterns observed in humans: monophasic, biphasic, and polyphasic $[14,15]$. Monophasic is defined as one 6-8 hour sleep cycle. Biphasic sleep is defined as one 5-7 hour sleep interval with one 30-minute nap time. Lastly, polyphasic sleep is characterized by one 5-6 hour sleep interval with a minimum of two 30-minute nap times (Figure 2). Current research has not yet explored the correlation between different sleeping patterns and glymphatic clearance relative to $\mathrm{A} \beta$ accumulation and $\mathrm{AD}$. Identifying an optimal sleep pattern that may reduce $A \beta$ accumulation could slow down the cognitive decline in individuals with AD.

\section{Different Sleep Cycles}

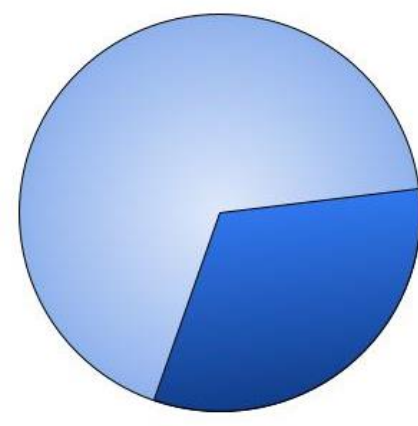

Monophasic

$16-8$ hours

Wake

Sleep

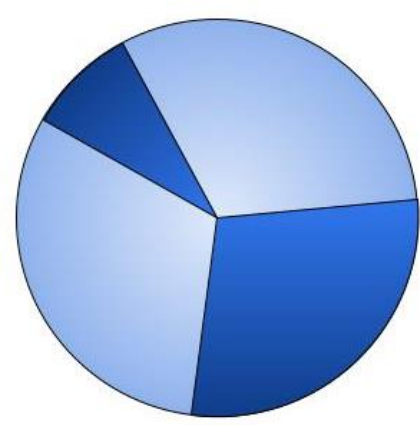

Biphasic

1 5-7 hours

$1.5-1 \mathrm{hr}$ nap

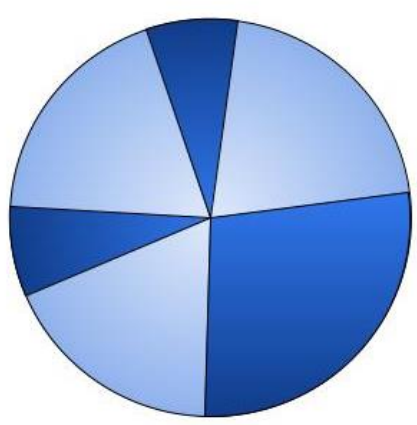

$$
\begin{gathered}
\text { Polyphasic } \\
1 \text { 5-7 hours } \\
2+.5-1 \text { hr nap }
\end{gathered}
$$

Figure 2. Sleep Patterns Across 24 hours. This figure was created using Biorender.

Slow-wave energy is the accumulated electroencephalography (EEG) oscillatory activity in the delta frequency band ( 1-4 Hz). Slow-wave sleep (SWS) or deep sleep, defined as Stage 3 of the sleep cycle, occurs during non-REM and is characterized by delta waves. During this stage, there is a decrease in blood pressure and breathing rate as the body repairs itself and consolidates memory. In other words, SWS is the stage needed for the body to feel reenergized. In contrast, the rapid eye movement (REM) sleep phase is what is known as paradoxical sleep. During this stage, oscillatory activity shows desynchronized rhythms most similar to arousal or wakefulness. REM sleep causes the muscles to become immobilized, dreaming occurs, and similar to SWS, memories are consolidated. As the glymphatic system has been suggested to work best during rested sleep, we propose that increased slow-wave sleep will lead to more glymphatic clearance. Monophasic sleep has been proposed to have more SWS than polyphasic sleep [16].

The glymphatic system, which plays a role in clearing $\mathrm{A} \beta$ buildup during sleep is important to study in its relation to AD. The bidirectional relationship between sleep and neurodegeneration makes it an important focal point for preventing the worsening of the disease state. As sleep is a malleable daily process, we hope the conclusions of this study will provide healthcare personnel with supporting rationale to integrate changes in sleep processes into $A D$ treatment plans. Understanding the connection between sleep patterns and the glymphatic system clearance in individuals with a family history of AD may also serve to promote better sleeping habits in order to reduce $A B$ accumulation especially for those who are at risk for the disease. Further, determining the optimal sleep patterns for glymphatic clearance could be used as a long-term lifestyle change to slow down or prevent disease progression especially in those with a family history of AD.

In this paper, we aim to provide an ad-hoc methodology to investigate the optimal sleeping pattern for the prevention of $\mathrm{AD}$. We hypothesize that there is a bidirectional relationship between $A B$ accumulation and lack of sleep. Bidirectionality between each factor has been theorized but has not yet been examined. In this study, we hypothesized that participants who engage in greater 
UNDERGRADUATE RESEARCH IN NATURAL AND CLINICAL SCIENCE AND TECHNOLOGY (URNCST) JOURNAL

Read more URNCST Journal articles and submit your own today at: https://www.urncst.com

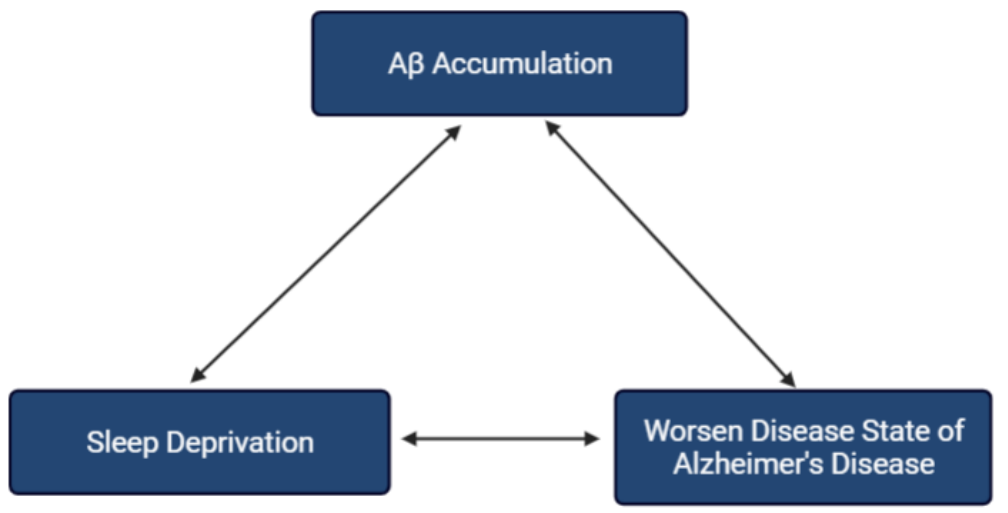

Figure 3. Concept model showing bidirectionality between $A ß$ accumulation, lack of sleep, and worsened disease state of AD. This figure was created using Biorender.

(1)

Enrollment

(2)

Pre-Assessment

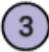

Observational Study

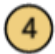

Post- Assessment

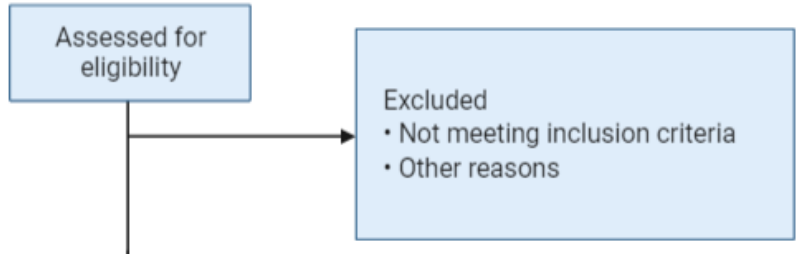

- Self Reported Life Style Questionnaire

- Self Reported Sleep Quality

- Inital PET Scans for measuring basal A $\beta$ accumulation, Overnight Sleep Study for SWS measurement, Multiple Sleep Latency Test

- Basal Memory and Cortisol measurements

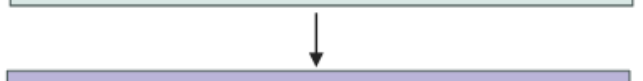

- Quantitative Sleep Measurement via Actigraphy

- Qualitiative Sleep, and Energy level

Measurement via Sleep Diary

- Blood Tests to measure cortisol and A $\beta$ levels

and memory tests (Every two weeks)

- Interim Overnight Sleep Study for SWS

measurement and Multiple Sleep Latency Test

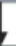

- Post Study Self Reported Life Style

Questionnaire

- Final PET Scans for measuring basal A $\beta$ accumulation, Overnight Sleep Study for SWS measurement, Multiple Sleep Latency Test

- Final Memory and Cortisol measurements

Figure 4. Diagram summarizing research design. This figure was created using Biorender. This ad-hoc cross-sectional study is designed to collect pertinent variables to understand the impact that sleep cycles have on the accumulation of $A B$ and cognitive decline in middle-aged adults. 
UNDERGRADUATE RESEARCH IN NATURAL AND CLINICAL SCIENCE AND TECHNOLOGY (URNCST) JOURNAL Read more URNCST Journal articles and submit your own today at: https://www.urncst.com

monophasic sleep will show fewer signs of $A \beta$ protein buildup and over time, slower rates of neurodegeneration, relative to those who engage in either biphasic and polyphasic sleep (Figure 3).

\section{Methods \\ Participant Enrollment}

The target group of this study will be adults between the ages 20-40 and 41-60 including both sexes to account for variability in sleeping patterns. Signs of late-onset AD begin around age 60 and signs of early-onset AD begin to show between ages 30 to 60 (Figure 4). Voluntary participants will be selected based on reported family history of AD. Participants will be excluded if they meet any of the following criteria: past/present medical history of cancer, psychiatric disorders, sleep disorders, those who are pregnant or breastfeeding, shift workers, or plan to travel outside of their time zone during the study period.

Prior to the beginning of the study, questionnaires administered by interviewers will collect information regarding participants' personal data, education, work, health status, and sleep patterns. After determining participants have met the criteria for participation, they will be asked to consent to participation via a written informed consent form. Participants will be assigned to one of three predetermined groups depending on their pre-enrollment sleeping pattern: monophasic, biphasic and polyphasic [17]. Participants will not need to adjust their sleep pattern to be included in the study, accounting for variability in adjustment time. Sleep quality over the past month will be calculated based on the Pittsburgh Sleep Quality Index.

\section{Schedule of Assessments}

Participants will be asked to have their sleeping patterns monitored using an actigraphy watch and a sleep diary for 6 months. Every day participants will be asked to record their subjective sleep levels and energy ratings in a journal entry. Participants will be asked to take a positron emission tomography (PET) scan to measure A $\beta$ plaque accumulation at the beginning and end of the study (months 0 and 6). Participants will be asked to perform a formal sleep study where polysomnograms and sleep staging will be calculated overnight followed by an MSLT study at the beginning, interim and conclusion of the study (months 0 , $3,6)$. Every two weeks a memory test will be conducted and blood work will be taken to measure cortisol and $A B$ levels.

\section{$\underline{\text { Measures }}$}

Memory

Sleep has been suggested to be important for declarative memory consolidation. A study even found that lower recognition of items learned previously was associated with lower subjective sleep quality in individuals with prodromal AD [18]. The present study opted to use memory recall test which involves the participants being given a set of words to memorize before sleeping and asking the participants to recall any words they remember the morning after the sleep cycle. The words will be given on a piece of paper in list form. Participants will be asked to only reference the list of words before sleeping for 5 minutes. Declarative memory tapping verbal learning and pattern recognition memory will be used. This test would be given every two weeks. The number of words correctly identified will be recorded.

\section{Sleep Diary}

Sleep has also been suggested to play a role in conserving and restoring energy through glycogen level restoration [19]. Therefore, testing energy levels may be indicative of sleep quality. Self-reported qualitative energy levels would be recorded daily by the participants through a sleep diary (Consensus Sleep Dairy) [20]. The quantitative energy levels will be recorded independently by each participant in a journal-style entry, ranking their energy level on a Likert-type scale of 1 (no energy) to 10 (full energy).

\section{Slow Wave Sleep (SWS) Measurement through EEG Analysis}

Polysomnograms and sleep staging will be calculated according to standard criteria using a a MK3 TrackIT recorder (Lifelines Diagnostics) [21]. Polysomnograms will be performed using standard electrodes including bilateral frontal, central and occipital parasagittal electrodes, and a 2-lead electrocardiogram, SWS will be quantified as the spectral power in the delta $(0.5-4 \mathrm{~Hz})$ band averaged across all non-REM epochs from the bilateral frontal and central electrodes. All data will be accurately collected and calculated by a registered polysomnographic technologist.

\section{Sleep Latency}

Sleep latency, or how fast one falls asleep, is used routinely as an indicator of pathological sleepiness. A sleep latency that is too short or too long would be indicative of sleep abnormalities whereas a normal sleep latency would indicate healthy sleep habits. One study suggested that a sleep latency of less than 5 minutes is associated with impaired performance while a sleep latency of 8 minutes or above is associated with a diagnosis of narcolepsy [22]. In this study, we define sleep as a regulated state of reduced movement and sensory responsiveness. Sleep latency will be quantitatively measured using the Multiple Sleep Latency Test (MSLT). The MSLT will consist of participants being asked to take 4-5 20-minute naps over a 2-hour period with EEG monitoring to determine sleep latency.

\section{Actigraphy Watch}

Actigraphy watch (MotionWatch 8) is an actigraphy device that will be used in this study. Measurements collected will include total sleep time, sleep efficiency, and 
UNDERGRADUATE RESEARCH IN NATURAL AND CLINICAL SCIENCE AND TECHNOLOGY (URNCST) JOURNAL Read more URNCST Journal articles and submit your own today at: https://www.urncst.com

circadian rhythm analysis. Individuals will be asked to wear the watch $24 / 7$ for the length of the study ( 6 months). The actigraphy watch will mainly be used to compare sleep quality between the three experimental groups. The actigraphy data will be manually scored using the 30 -second epoch timer stamps. Subjective bedtime and waketime scores will be set when the participant pushes a button on the actigraph indicated they have lain down with the intention to sleep and when they have woken, respectively. If the button is not pushes, the sleep diary, which contains written intended bedtime and sleep time will be used.

\section{Amyloid-Beta Buildup}

A $\beta$ Plaque buildup will be measured in two separate ways: through $\mathrm{C} 2 \mathrm{~N}$ Blood Testing and PET scanning. Blood samples collected from participants every two weeks will be tested using the $\mathrm{C} 2 \mathrm{~N}$ test (PrecivityAD) that uses mass spectrometry to detect $A \beta$ levels. $A \beta$ levels calculated through blood testing will be used to identify interim variations in $A \beta$ levels through the study. Amyloid-beta burden $(\mathrm{A} \beta \mathrm{B})$ levels would be measured using PET. This will be done using 8F- florbetaben (FBB), a PET radiotracer. Levels of $A \beta$, calculated through both $C 2 N$ testing and PET scans will be compared to their baseline $\mathrm{A} \beta \mathrm{B}$ levels at the end of the study.

\section{Cortisol}

Cortisol levels will be measured using blood tests taken every two weeks during the study to account for any variation in quality of sleep that may affect results. In addition, SWS is associated with decreased plasma cortisol levels. Therefore, measuring cortisol levels may allow us to determine whether participants demonstrated more SWS in each sleep pattern pool.

\section{Analysis}

PET scan data on $A B$ accumulation will be used to determine an overall increase or decrease of $A ß B$ from the start to the finish of the study, whereas, $A B$ levels calculated through blood samples will be used to calculate interim changes in the protein level. Data collected from the actigraph will be used to measure both qualitative (Consensus Sleep Diary) and quantitative (energy levels) measures of sleep. Data collected from the polysomnographs will be used to determine increases and decreases in SWS. Daily energy levels determine how energy levels changed throughout the course of the study, determine if there was any improvement, and compare averages between each sleep group. Individual results of each sleep group will be averaged out and compared to determine patterns of accumulation and other variables. Data on cortisol levels were collected to control for variability across the following measures: AB levels, memory test scores, and data from actigraphy watch on hours of sleep between individuals. All analysis will be conducted using the SAM data analytics approach to better identify correlations between the measures and combine to test the hypothesis offered [23].

\section{Results}

We expect $A \beta$ protein accumulation (from PET scans) and cortisol levels to decreaseand SWS activity to increase the most in participants in the monophasic sleep group followed by participants in the polyphasic sleep group. Lastly, the biphasic sleep group should show the least improvement in metabolic levels. This is because participants in the polyphasic sleep group will on average sleep more with two nap times rather than one and therefore have increased SWS. Additionally, we expect higher cognitive scores on the memory test, increased energy ratings, and normal sleep latency in participants in the monophasic sleep group followed by the polyphasic sleep group and lastly the biphasic sleep group.

\section{Discussion}

This research proposes to test the effects of different sleeping patterns particularly monophasic, biphasic, and polyphasic sleep in relation to glymphatic clearance. By taking periodic tests of $\mathrm{A} ß$ levels using a large population of individuals with a family history of $\mathrm{AD}$, optimal sleeping patterns can be determined. The results of this study can be applied to a larger population of older adults with AD and implications can inform preventative measures for $\mathrm{AD}$ or even as part of a treatment plan. Individuals who have a family history of AD or who are identified as high risk for $\mathrm{AD}$ may be recommended to adopt monophasic sleep. Some clinical methods to improve sleep cycles, such as stimulus control therapy, sleep restriction, or the use of oral medication such as melatonin, can be discussed with the patient as an addition to their current treatment plan. In addition, determining the sleeping pattern that has the least $\mathrm{A} \beta$ clearance can allow clinicians to use this as a prognosis tool for $\mathrm{AD}$ by identifying which individuals are more likely to have decreased $A B$ clearance, therefore, are most at risk for $\mathrm{AD}$.

To our knowledge, research connecting glymphatic clearance and sleep patterns have not been conducted yet. Therefore, we suggest that if significant effects of monophasic sleep patterns on neurodegeneration are found, it may be beneficial to those who are predisposed to AD to alter their sleep pattern as part of their management of the disease. In this paper, we anticipate that the participants with the most $A B$ clearance would be those with a monophasic sleep pattern. Aß protein accumulation and increased metabolic waste levels, which are characterized by an enlargement of the perivascular space, may show signs of neurodegeneration in the PET brain scans. Past research indicates that increased slow-wave activity after sleep deprivation is a normal response due to increased homeostatic sleep pressure. Split sleep (polyphasic/biphasic) reduces slow-wave activity and 
UNDERGRADUATE RESEARCH IN NATURAL AND CLINICAL SCIENCE AND TECHNOLOGY (URNCST) JOURNAL Read more URNCST Journal articles and submit your own today at: https://www.urncst.com

therefore homeostatic sleep pressure. Slow-wave sleep has also been linked to increased glymphatic system clearance. For this reason, we propose that the glymphatic system functions more efficiently during SWS and therefore reduced SWS would lead to disrupted glymphatic clearance and increased disease progression.

To measure $A B$ accumulation and determine optimal sleeping patterns, radiotracers and blood tests will be conducted. One limitation includes the inability of these radiotracers to measure soluble $A \beta$ and therefore, possible inaccurate results that do not cover the clearance of soluble $A ß$. Since this is an ad-hoc study, outside variables including slight variations in sleep pattern, environment, location, varying job schedules, etc. cannot be accounted for. However, to help mitigate this, a survey will be used to group similar participants, an actigraphy watch will be used to detect slight differences in sleep pattern, and a large sample will be used. For future directions, studies show that those who live on the equator are more likely to partake in polyphasic sleep due to a hotter environment. Therefore, it may be valuable to investigate the correlation of sleep patterns with glymphatic clearance of those who live near the equator or those who originate from countries near the equator. Such a study would need to account for differences in environment and location.

\section{Conclusions}

This study explores the connection between $A B$ deposition, the glymphatic system and sleep patterns by proposing a method for an ad-hoc sleep study. Future studies may investigate the connection between sleep cycles and glymphatic system clearance to further understand the effects of different types of sleep on AD. The population used for this study includes individuals with a family history of AD. Genetic tests of the participants can be collected at the end of the sleep study to identify if there is a stronger relationship between $A B$ clearance with those who have both a family history of AD and carry causative gene markers. Further research can focus on an older adult population who have been diagnosed with $\mathrm{AD}$ to understand if shifting to a monophasic sleep pattern could potentially slow $A B$ accumulation. Determining optimal sleep patterns for $A B$ clearance in those with a family history of $\mathrm{AD}$ would allow the option for this population to adopt sleeping habits that closely resemble monophasic sleep. This proposal can be used as a preventative measure to slow or prevent disease progression even early on in life.

\section{List of Abbreviations Used}

AD: Alzheimer's disease

CNS: central nervous system

Aß: amyloid-beta

APP: amyloid-beta precursor protein

Aßb: amyloid-beta burden

FBB: 8F- florbetaben

CSF: cerebrospinal fluid
PET: positron emission tomography

SWS: slow-wave sleep

REM: rapid eye movement

ISF: interstitial fluid

AQP4: aquaporin - 4

EEG: electroencephalography

MSLT: multiple sleep latency test

\section{Conflicts of Interest}

The authors declare no conflicts of interest.

\section{Ethics Approval and/or Participant Consent}

Study participants will sign a detailed consent form to measure and collect quantitative and qualitative sleep measures, cortisol and $A B$ levels during the 6-month trial. Participants will not be asked to alter their sleeping and daily habits as this is an observational study. Participants will also be asked to complete a survey prior to being selected, to determine eligibility. Surveys to collect qualitative measures throughout and post-study will be given to participants. No Research Ethics Board (REB) review was required as this paper is a proposal, not primary research. This study needs to be approved by The Health Canada and Public Health Agency of Canada (PHAC) REB prior to beginning the study.

\section{Authors' Contributions}

MB: contributed to the design of the study, collected and analysed data, drafted the manuscript, created all of the figures, and gave final approval of the version to be published.

SM: contributed to the design of the study, collected and analysed data, drafted the manuscript, and gave final approval of the version to be published.

MM: contributed to the design of the study, collected and analysed data, drafted the manuscript, and gave final approval of the version to be published.

\section{Acknowledgements}

We would like to extend our gratitude to Elly Wong for her mentorship, support, and advice through the process of this paper. We would also like to thank the URNCST team for organizing the mentored paper initiative.

\section{Funding}

This study was not funded

\section{References}

[1] Abbott SM, Videnovic A. Chronic sleep disturbance and neural injury: links to neurodegenerative disease. Nature and Science of Sleep. 2016 Jan 25;8:55-61. https://doi.org/10.2147/NSS.S78947

[2] Ju YES, Lucey BP, Holtzman DM. Sleep and Alzheimer disease pathology - A bidirectional relationship. Nature Reviews Neurology. 2013 Dec 24;10(2):115-119. https://doi.org/10.1038/nrneurol.2013.269 
UNDERGRADUATE RESEARCH IN NATURAL AND CLINICAL SCIENCE AND TECHNOLOGY (URNCST) JOURNAL

Read more URNCST Journal articles and submit your own today at: https://www.urncst.com

[3] Moran M, Lynch C, Walsh C, Coen R, Coakley D, Lawlor B. Sleep disturbance in mild to moderate Alzheimer's disease. Sleep Medicine. 2005 July;6(4):347-352. https://doi.org/10.1016/j.sleep.2004 .12 .005

[4] Xie L, Kang H, Xu Q, Chen MJ, Liao Y, Thiyagarajan M, O’Donnell J, Christensen DJ, Nicholson C, Iliff JJ, Takano T, Deane R, Nedergaard M. Sleep drives metabolite clearance from the adult brain. Science. 2013 Oct 18;342(6156):373-377. https://doi.org/10.1126/ science.1241224

[5] Underwood E. Sleep: The brain's housekeeper? Science (American Association for the Advancement of Science). 2013 Oct 18;342(6156):301-301. https://doi.org/10.1126/science.342.6156.301

[6] Saper CB, Scammell TE, Lu J. Hypothalamic regulation of sleep and circadian rhythms. Nature. 2005 Oct 27;437 (7063):1257-1263. https://doi.org/10.1038/nature04284

[7] Nedergaard M. Garbage truck of the brain. Science (American Association for the Advancement of Science). 2013 June 28;340(6140):1529-1530. https://doi.org/10.1126/science.1240514

[8] Benveniste H, Liu X, Koundal S, Sanggaard S, Lee H, Wardlaw J. The glymphatic system and waste clearance with brain aging: A review. Gerontology (Basel). 2019;65(2):106-119. https://doi.org/10.1159/ 000490349

[9] Jessen NA, Munk ASF, Lundgaard I, Nedergaard M. The glymphatic system: A beginner's guide. Neurochemical Research. 2015 May 7;40(12):25832599. https://doi.org/10.1007/s11064-015-1581-6

[10] O'Brien RJ, Wong PC. Amyloid precursor protein processing and Alzheimer's disease. Annual Review of Neuroscience. 2011 Mar 29;34(1):185-204. https://doi.org/10.1146/annurev-neuro-061010-113613

[11] Kang JE, Lim MM, Bateman RJ, Lee JJ, Smyth LP, Cirrito JR, Fujiki N, Nishino S, Holtzman DM. Amyloid- $\beta$ dynamics are regulated by orexin and the sleep-wake cycle. Science (American Association for the Advancement of Science). 2009 Nov 13;326(5955): 1005-1007. https://doi.org/10.1126/science.1180962

[12] Roh JH, Jiang H, Finn MB, Stewart FR, Mahan TE, Cirrito JR, Heda A, Snider BJ, Li M, Yanagisawa M, de Lecea L, Holtzman DM. Potential role of orexin and sleep modulation in the pathogenesis of Alzheimer's disease. The Journal of Experimental Medicine. 2015;212(1):121-121. https://doi.org/10.1084/jem.20141 $78812122014 \mathrm{c}$

[13] Ekirch AR. Segmented sleep in preindustrial societies. Sleep. 2016 Mar 1;39(3):715-716. https://doi.org/ $\underline{10.5665 / \text { sleep. } .5558}$
[14] Stranges S, Tigbe W, Gómez-Olivé FX, Thorogood M, Kandala NB. Sleep problems: An emerging global epidemic? Findings from the INDEPTH WHO-SAGE study among more than 40,000 older adults from 8 countries across Africa and Asia. Sleep. 2012;35:11731181. https://doi.org/10.5665/sleep

[15] Saeed Z, Hasan Z, Atif M. Sleep patterns of medical students: Their relationship with academic performance: A cross sectional survey. Professional Medical Journal. 2015;22:913-923. https://doi.org/10.29309/TPMJ/2015 .22 .07 .1183

[16] Fagioli I, Salzarulo P. Dynamics of EEG background activity level during quiet sleep in multiple nocturnal sleep episodes in infants. Electroencephalography and Clinical Neurophysiology. 1997 Dec;103(6):621-626. https://doi.org/10.1016/S0013-4694(97)00076-X

[17] Al-Abri MA, Al Lawati I, Zadjali F, Ganguly S. Sleep patterns and quality in Omani adults. Nature and Science of Sleep. 2020 Apr 14;12:231-237. https://doi.org/10.2147/NSS.S233912

[18] Westerberg CE, Lundgren EM, Florczak SM, Mesulam MM, Weintraub S, ZEE PC, Paller KA. Sleep influences the severity of memory disruption in amnestic mild cognitive impairment: Results from sleep self-assessment and continuous activity monitoring. Alzheimer Disease and Associated Disorders. 2010 Oct-Dec;24(4):325-333. https://doi.org/10.1097/WAD.0b013e3181e30846

[19] Bak LK, Walls AB, Schousboe A, Waagepetersen HS. Astrocytic glycogen metabolism in the healthy and diseased brain. The Journal of Biological Chemistry. 2018 Mar 23;293(19):7108-7116. https://doi.org/ $\underline{10.1074 / j b c . R 117.803239}$

[20] Carney CE, Buysse DJ, Ancoli-Isreal S, Edinger JD, Krystal AD, Lichstein KL, Morin CM. The consensus sleep diary: Standardizing prospective sleep selfmonitoring. Sleep. 2021 Feb 1;35(2):287-302. https://doi.org/10.5665/sleep.1642

[21] Iber C. The AASM manual for the scoring of sleep and associated events: Rules, terminology and technical specifications. American Academy of Sleep Medicine. 2007. Available from: https://www.sleep.pitt.edu/wpcontent/uploads/2020/03/The-AASM-Manual-forScoring-of-Sleep-and-Associated-Events-2007-.pdf

[22] Thomas D. Multiple sleep latency test (MSLT). In Encyclopedia of sleep. 2013:96-99. https://doi.org/ 10.1016/B978-0-12-378610-4.00146-7

[23] Cheung ML, Jak S. Analyzing big data in psychology: A split/analyze/ meta-analyze approach. Frontier Psychology. 2016 May 23. https://doi.org/10.3389/fpyg .2016 .00738 
UNDERGRADUATE RESEARCH IN NATURAL AND CLINICAL SCIENCE AND TECHNOLOGY (URNCST) JOURNAL

Read more URNCST Journal articles and submit your own today at: https://www.urncst.com

\section{Article Information}

Managing Editor: Jeremy Y. Ng

Peer Reviewers: Elly Wong, Ricky Chow

Article Dates: Received Sep 04 21; Accepted Oct 20 21; Published Nov 1921

\section{Citation}

Please cite this article as follows:

Bansal M, Musa SS, Mulpuru M. Determining the optimal sleep pattern to promote glymphatic clearance of amyloid-beta in individuals at risk for Alzheimer's disease: A research protocol. URNCST Journal. 2021 Nov 19: 5(11).

https://urncst.com/index.php/urncst/article/view/324

DOI Link: https://doi.org/10.26685/urncst.324

\section{Copyright}

(c) Muskan Bansal, Shahad S. Musa, Manitha Mulpuru. (2021). Published first in the Undergraduate Research in Natural and Clinical Science and Technology (URNCST) Journal. This is an open access article distributed under the terms of the Creative Commons Attribution License (https://creativecommons.org/licenses/by/4.0/), which permits unrestricted use, distribution, and reproduction in any medium, provided the original work, first published in the Undergraduate Research in Natural and Clinical Science and Technology (URNCST) Journal, is properly cited. The complete bibliographic information, a link to the original publication on http://www.urncst.com, as well as this copyright and license information must be included.

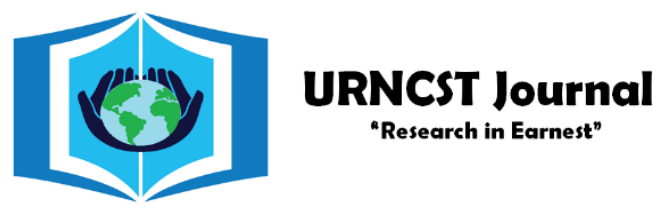

\section{Funded by the Government of Canada}

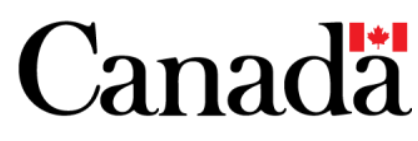

Do you research in earnest? Submit your next undergraduate research article to the URNCST Journal!

| Open Access | Peer-Reviewed | Rapid Turnaround Time | International |

| Broad and Multidisciplinary | Indexed | Innovative | Social Media Promoted |

Pre-submission inquiries? Send us an email at info@ urncst.com | Facebook, Twitter and LinkedIn: @URNCST

Submit YOUR manuscript today at https://www.urncst.com! 